\title{
Hamiltonian Approach to the Gribov Problem
}

\author{
T. Heinz \\ anstitut für Theoretische Physik, Universität Regensburg, D-93040 Regensburg, Germany
}

\begin{abstract}
We study the Gribov problem within a Hamiltonian formulation of pure Yang-Mills theory. For a particular gauge fixing, a finite volume modification of the axial gauge, we find an exact characterization of the space of gauge-inequivalent field configurations.
\end{abstract}

\section{Introduction}

It is well known that the main problem for a quantum formulation of gauge theories is the fact that the space $\mathcal{A}$ of gauge potentials is 'too large'; there are infinitely many gauge equivalent configurations corresponding to one and the same physical situation. This huge redundancy should be eliminated by constructing the physical configuration space, the space of orbits, $\mathcal{A}_{\text {phys }}=\mathcal{A} / \mathcal{G}$, consisting of gauge fields modulo gauge transformations, the elements of the gauge group $\mathcal{G}$.

The aim of this contribution is to find the physical configuration space $\mathcal{A}_{\text {phys }}$ by identifying a subset of $\mathcal{A}$ with $\mathcal{A}_{\text {phys }}$. The method for doing that is the familiar procedure of gauge fixing, which amounts to choosing a representative gauge potential $A$ on each of the orbits. In doing that, two criteria have to be met.

(i) existence: A representative should be selected on any orbit; no orbit should be omitted.

(ii) uniqueness: There should be only one representative obeying the gauge condition on each orbit. If, on the other hand, there are (at least) two gauge equivalent fields, $A_{1}, A_{2}$, satisfying the gauge condition, the gauge is not completely fixed. There is a residual gauge freedom given by a gauge transformation, say, $U$, between the 'Gribov copies' [1], $U: A_{1} \rightarrow A_{2}$.

In view of that, it is clear that the physical configuration space $\mathcal{A}_{\text {phys }}$ is the maximal subset of $\mathcal{A}$ containing no Gribov copies. It is called a 'fundamental modular domain' [2].

In order to find the physical configuration space we follow the pioneering work of Feynman

\footnotetext{
*e-mail: thomas.heinzl@physik.uni-regensburg.de
}

on (2+1)-dimensional Yang-Mills theory [3] and make use of the intuitive Hamiltonian formalism within a functional Schrödinger picture. As reemphasized recently in [4], there are reasons to believe that the fundamental domain (at least in $2+1$ dimensions) has a finite volume leading to a purely discrete spectrum. This would provide a natural explanation of a mass gap in the theory. The quantum mechanical analogue for this is the infinite square well of extension $d$. The gap between the ground state and the first excited state is of the order $1 / d^{2}$. The non-abelian case corresponds to finite $d$ and thus to a finite gap; the abelian case corresponds to infinite size, $d \rightarrow \infty$, and thus to vanishing gap (the analogue of the massless photon).

\section{An Example from Quantum Mechanics}

To gain some more intuition let us stay a little bit further within the context of quantum mechanics. Consider a point particle moving in a plane described by (cartesian) coordinates $q_{1}, q_{2}$, and let the particle have vanishing angular momentum, $G \equiv q_{1} p_{2}-q_{2} p_{1}=0$. This latter identity we interpret as Gauss's law, so that $G$ is the generator of gauge transformations which are just rotations around the origin. If we introduce polar coordinates, the radius $r$, and the angle $\phi$, it is obvious that the angle changes under rotations and is thus gauge variant, whereas the radius $r$ is the gauge invariant variable. Accordingly, the physical configuration space is the positive real line. The associated physical Hamiltonian is

$$
H_{\text {phys }}=-\frac{1}{2} r^{-1} \frac{\partial}{\partial r} r \frac{\partial}{\partial r}
$$


and depends only on the gauge invariant variable $r$, as it should.

Let us assume now that we are not as smart as to guess the gauge invariant variables and proceed in a pedestrian's manner via gauge fixing. We gauge away $q_{2}, \chi(q) \equiv q_{2}=0$, and immediately realize that this gauge choice selects two representatives on each orbit at $\pm q_{1}$ (see Fig. 1). There is a (discrete) residual gauge freedom between the copies, $q_{1} \rightarrow-q_{1}$. So we have a Gribov problem, which is best analysed in terms of the Faddeev-Popov (FP) operator [5] given by the commutator of the gauge fixing with Gauss's law,

$\mathrm{FP} \equiv-i[\chi, G]=q_{1}$

The latter turns out to be coordinate dependent and vanishes at the 'Gribov horizon', $q_{1}=0$, which is just the point separating the two gauge equivalent regions $q_{1}>0$ and $q_{1}<0$. So we can fix the gauge completely by demanding that $q_{1}$ be positive (an inequality, thus a non-holonomic constraint), and thus again we find that the physical configuration space is the positive real line where we can identify $q_{1}$ with the radius $r$. Due to the simplicity of the example the decomposition of the configuration space into its redundant and physical parts via gauge fixing can easily be visualized (Fig. 1).

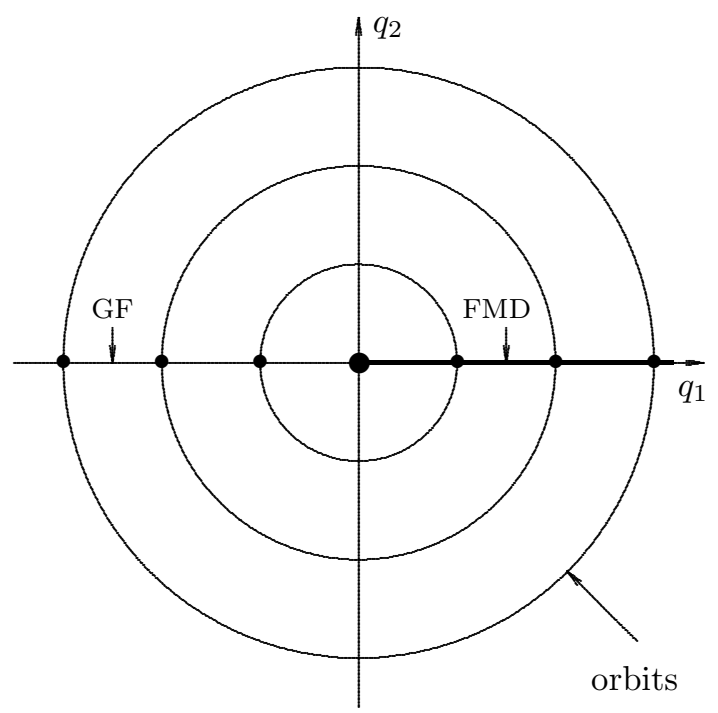

Figure 1: The configuration space of the quantum mechanical example. The orbits are circles around the origin. The gauge fixing $(G F), q_{2}=0$, cuts every orbit twice at the Gribov copies $\pm q_{1}$ $(\bullet)$. The Gribov horizon is the origin, the fundamental modular domain (FMD) the positive real line, $q_{1}>0$.

Using Gauss's law to eliminate the unphysical momentum, $p_{2}$, from the original Hamiltonian, $H=\left(p_{1}^{2}+p_{2}^{2}\right) / 2$, one ends up with the physical Hamiltonian (11). Note the additional factors of $r=|\operatorname{det} \mathrm{FP}|$ in the kinetic term. They guarantee that the Hamiltonian is hermitean on $\mathcal{L}^{2}\left(r d r, \mathcal{R}^{+}\right)$.

As a result we can state that gauge fixing amounts to mapping the original cartesian coordinates onto curvilinear ones and setting the gauge variant part of these (the 'angles') to zero. The Jacobian of the transformation is the FP determinant and enters the physical Hamiltonian as well as the scalar product [6].

\section{Yang-Mills Theory in Palumbo Gauge}

Let us now consider the case of field theory. According to Dirac, there is still another, more physical, interpretation of gauge fixing as a 'dressing' of the fermions |7|. In the abelian case, one has the dressed electron

$\psi_{\chi}(\mathbf{x})=\psi(\mathbf{x}) \exp i e \int d^{3} y \mathbf{E}_{\chi}(\mathbf{x}-\mathbf{y}) \cdot \mathbf{A}(\mathbf{y})$,

where $\mathbf{E}_{\chi}$ is the dressing electric field corresponding to the gauge fixing $\chi=0$. For the Coulomb gauge it is of course the familiar Coulomb field [7], for the axial gauge, $\chi \equiv A_{3}=0$, it is the singular configuration

$E_{1}=E_{2}=0, \quad E_{3}=e \theta\left(x_{3}\right) \delta\left(x_{1}\right) \delta\left(x_{2}\right)$,

corresponding to a string of electric flux in the 3 -direction [7]. It is easy to check that (11) is a singular solution of Gauss's law for a point charge at the origin,

$G \equiv \partial_{i} E_{i}=e \delta^{3}(\mathbf{x})=e \rho_{\perp}\left(x_{1}, x_{2}\right) \rho_{L}\left(x_{3}\right)$,

where the last step stands for a smearing of the delta functions in transverse and longitudinal direction with respect to the string. It has been 
known for a long time that the singular configuration (4) leads to an infrared divergence in the energy [0] of the form

$H \sim e L\left[\int d x_{3} \rho_{L}\left(x_{3}\right)\right]^{2}+$ finite,

where we have introduced the length $L$ of the string. The remedy of this infrared problem is to introduce a homogeneous background charge density in 3-direction, $\rho_{L} \equiv \delta\left(x_{3}\right)-1 / 2 L$. Calculating the integral of the Gauss operator, $G$,

$$
\int_{-L}^{L} d x_{3} G \sim \int_{-L}^{L} d x_{3} \rho_{L}=0,
$$

one finds that the zero mode of $G$ is the charge of the string which vanishes, and that the energy of the string is finite. Thus, only neutral strings have finite energy, a property somewhat reminiscent of confinement. Due to the manifest appearance of (chromo-)electric strings, the axial gauge has been suggested as an appropriate gauge choice for studying the confinement problem [9].

There are several gauges which correspond to the above modification of the axial gauge (differing in the way the residual gauge freedom is fixed (10). However, all of them have in common that $A_{3}$ is not completely set to zero, but only those modes with momentum $k_{3} \neq 0$. A zero mode, having $k_{3}=0$, is retained,

$$
A_{3}\left(x_{1}, x_{2}, x_{3}\right) \rightarrow a_{3}\left(x_{1}, x_{2}\right) .
$$

To fix the residual gauge freedom still left one can do a (self-) similar construction in 1- and 2direction. This is the Palumbo gauge [11]. Details are unnecessary for what follows.

The gauge fixing (8) can be shown to exist by explicitly constructing the transformation $U$ that takes an arbitrary configuration to this gauge. One finds that $U$ is of the form $U=W \times V$, with

$$
\begin{aligned}
W(\mathbf{x}) & =P \exp i \int_{-L}^{x_{3}} d y_{3} A_{3}\left(x_{1}, x_{2}, y_{3}\right), \\
V(\mathbf{x}) & =\exp \left[i\left(x_{3}+L\right) a_{3}\left(x_{1}, x_{2}\right)\right] .
\end{aligned}
$$

The first exponential, $W$, takes one to the pure axial gauge $\left(A_{3} \rightarrow 0\right)$, the second exponential, $V$, restores the zero mode $\left(0 \rightarrow a_{3}\right)$, which is determined via $2 L a_{3}\left(x_{1}, x_{2}\right)=\log w\left(x_{1}, x_{2}\right)$, where $w\left(x_{1}, x_{2}\right) \equiv W\left(x_{1}, x_{2}, L\right)$. Note that $a_{3}$ is in the Lie algebra $s u(2)$ and thus obtained from the group element $W \in S U(2)$ by taking a logarithm. As the latter is a multivalued function we are right at the question of uniqueness.

The main virtue of the Palumbo gauge (and related gauges) is the fact that the FP operator, its inverse and its determinant can be calculated exactly [10. The result for the determinant is

$\operatorname{det} \mathrm{FP}=\frac{\sin ^{2}\left|a_{3}\right| L}{\left(\left|a_{3}\right| L\right)^{2}}$,

with $\left|a_{3}\right|=\left(a_{3}^{a} a_{3}^{a}\right)^{1 / 2}$ being the modulus of $a_{3}$. The FP determinant (11) is just the Haar measure of $S U(2)$, thus the Jacobian of the exponential map, exp : su(2) $\rightarrow S U(2)$, from the algebra to the group. At the Gribov horizons, where $\operatorname{det} \mathrm{FP}$ vanishes, one has $\left|a_{3}\right|=\pi k / L, k$ integer, (in the algebra) and $w= \pm 1$ (in the group). At these points, the exponential map becomes singular, the inverse map, the logarithm, becomes multi-valued and thus the 'angle' variable $a_{3}$ illdefined. If one writes the zero mode with the help of a unit vector $n^{a}$ in color space as $a_{3}^{a}=\left|a_{3}\right| n^{a}$, the residual gauge freedom can be described in the following way: one has transformations $u_{1}(k)$ which do not change the direction of the color vector

$u_{1}(k):\left|a_{3}\right| n^{a} \rightarrow\left|a_{3}+2 \pi k / L\right| n^{a}$.

The second type of residual gauge transformations, $u_{2}$, transforms along the Gribov horizons ( $w= \pm 1$ ) without changing the length of the color vector,

$u_{2}:\left|a_{3}\right| n^{a} \rightarrow\left|a_{3}\right| \tilde{n}^{a}, \quad\left|a_{3}\right|=\pi k / L$.

The fundamental modular domain is therefore given in terms of the inequality $\left|a_{3}\right|<\pi / L$, which describes the interior of a sphere in color space (see Fig. 2). 


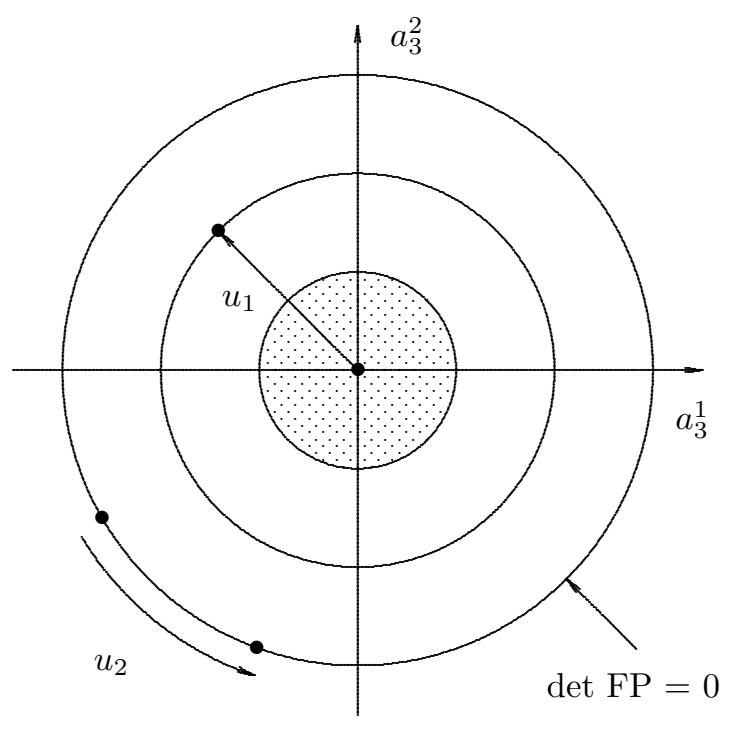

Figure 2: A two-dimensional plot of the configuration space of $a_{3}^{a}, a=1,2$. The Gribov horizons where det FP vanishes are (apart from the origin) circles of radius $\pi / L, 2 \pi / L$ etc. The shaded interior of the inner circle constitutes the fundamental modular domain. The arrows denote the action of the residual gauge transformations $u_{1}(k=1)$ and $u_{2}$ between particular Gribov copies represented by black dots $(\bullet)$.

The physical Hamiltonian containing all the Jacobian factors is a somewhat lengthy expression and can be found in [10].

One crucial question remains to be answered: what is the physics associated with the Gribov horizons being discontinuities in field space [12]? To find the answer one would need some geometrical space-time picture of the configurations at the horizon. In related gauges, they seem to correspond to monopoles 13 or magnetic vortices 14. An explicit but formal construction of a horizon configuration has been given in 15 .

The center of the group seems to play a peculiar role as the horizon configurations correspond exactly to group elements valued in the center, $w= \pm 1$. Finally, a $\theta$-angle might emerge when the wave functional $\Psi$ 'bites its tail' at the horizon with a quasi-periodicity like $\Psi(\pi / L)=$ $e^{i \theta} \Psi(-\pi / L)$.
It might be worthwhile to study these issues on a lattice where one has a finite number of degrees of freedom and a better control of infinities. In the context of maximally abelian gauge fixing on a lattice, the Gribov problem and its relation to the monopole condensation scenario of confinement have recently attracted a lot of attention [16]. A lattice gauge fixing similars to ours has just appeared in the literature 17]. It looks promising to investigate these issues further.

\section{Acknowledgements}

The author gratefully acknowledges instructive discussions with A. Jaramillo, S. Shabanov and D. Zwanziger. It is a pleasure to thank the organizer S. Narison and his team for all their efforts.

\section{REFERENCES}

[1] V.N. Gribov, Nucl. Phys. B139 (1978) 1

[2] D. Zwanziger, Nucl. Phys. B345 (1990) 461; P. van Baal, Nucl. Phys. B369 (1992) 259, and references therein

[3] R.P. Feynman, Nucl. Phys. B188 (1981) 479

[4] D. Karabali, V.P, Nair, Nucl. Phys. B464 (1996) 135

[5] L.D. Faddeev, V.N. Popov, Phys. Lett. B25 (1967) 29

[6] N. Christ, T.D. Lee, Phys. Rev. D22 (1980) 939; H. Cheng, E.-C. Tsai, Chin. J. Phys. 25 (1987) 95

[7] P.A.M. Dirac, Canad. J. Phys. 33 (1955) 650

[8] J. Schwinger, Phys. Rev. 130 (1963) 402

[9] S. Mandelstam, Phys. Rev. D19 (1979) 2391

[10] T. Heinzl, hep-th/9604018, and references therein

[11]F. Palumbo, Phys. Lett. B173 (1986) 81; B243 (1990) 109

[12]R. Jackiw, I. Muzinich, C. Rebbi, Phys. Rev. D17 (1978) 1576

[13] V.A. Franke, Yu. V. Novozhilov, E.V. Prokhvatilov, Lett. Math. Phys. 5 (1981) 239

[14] H.W. Grießhammer, hep-ph/9509417

[15] H.R. Petry, D. Schütte, Bonn preprint (1993)

[16] A. Hart, M. Teper, hep-lat/9606007, and references therein

[17] K. Bernstein, G. Di Cecio, W. Haymaker, hep-lat/9606018 